\title{
Destinação de cargas abandonadas e em processo de perdimento no Aeroporto de Internacional de Viracopos - aplicação da legislação ambiental pelo Ibama
}

Destination of abandoned cargos and in confiscation process in Viracopos International Airport - Environmental law application by Ibama

Asignación de cargas abandonadas y en proceso de confiscación en el aeropuerto internacional de Viracopos - Aplicación de la legislación ambiental por el Ibama

Luis Antonio Gonçalves de Lima Chefe da Unidade Avançada do Ibama no Aeroporto Internacional de Viracopos (UA-VCP), Campinas/SP, Brasil vcp.sp@ibama.gov.br

\section{Eloi Norberto Venturini Junior}

Chefe Substituto do Ibama UA-VCP, Campinas/SP, Brasil Mestrando - PPG em Engenharia Civil - Área de Saneamento e Ambiente Faculdade de Engenharia Civil, Arquitetura e Urbanismo (FEC), Universidade Estadual de Campinas (Unicamp), Campinas/SP, Brasil eventurini@gmail.com

Marcos José de Oliveira Analista Ambiental do Ibama UA-VCP, Campinas/SP, Brasil Doutorando - PPG Ciências da Engenharia Ambiental, Escola de Engenharia de São Carlos (EESC), Universidade de São Paulo (USP), São Carlos/SP, Brasil marcos.jose@gmail.com 


\title{
RESUMO
}

A quantidade de resíduos sólidos produzida em aeroportos equivale ao lixo gerado em cidades pequenas (de 5 a 30 mil habitantes), tornando o gerenciamento de resíduos uma das questões ambientais mais importantes em recintos aeroportuários. Além dos resíduos habituais gerados dentro dos aeroportos, uma importante (mas pouco conhecida) fonte de resíduos está associada a operações de comércio exterior: cargas abandonadas ou sujeitas à pena de perdimento. O objetivo do trabalho é relatar uma iniciativa, em execução pelo Ibama no Aeroporto Internacional de Viracopos (Campinas/SP), que consiste na aplicação da Política Nacional de Resíduos Sólidos (Lei no 12.305/2010) para a redução e correta destinação de resíduos sólidos relacionados a cargas abandonadas em aeroportos. Das cerca de 1.000 toneladas de cargas abandonadas em Viracopos, promoveu-se, em 2014 e 2015, a solução para 35.000 cargas (ou 103 ton.), reduzindo $10 \%$ do peso, $41 \%$ dos volumes e $33 \%$ dos conhecimentos aéreos, demonstrando que são expressivos e positivos os resultados das ações do Ibama. Além de beneficiar o depositário, ao liberar espaço útil nos terminais de cargas, a solução das cargas abandonadas implica na redução de danos ao Erário, assim como na redução de riscos de acidentes potencialmente danosos às pessoas e ao meio ambiente.

PALAVRAS-CHAVE: Resíduos sólidos. Gerenciamento. Política ambiental. Comércio exterior.

\begin{abstract}
The amount of solid waste produced in airports is equivalent to the waste generated in small cities (5-30 thousand inhabitants), making the waste management one of the most important environmental issues in airports sites. Besides the usual waste generated within the airports, an important (but little known) waste source is associated with international trade operations: abandoned cargos or in confiscation process (subject to penalty of loss). This work has the objective to report an initiative, under implementation by Ibama at the Viracopos International Airport (Campinas/SP/Brazil), which comprises applying the National Policy on Solid Waste (Brazilian Law No. 12.305/2010) for the reduction and proper disposal of solid waste related to abandoned cargos at airports. Of the approximately 1,000 tons of abandoned cargos in Viracopos, was promoted, in 2014 and 2015, the solution to 35,000 cargos (or 103 ton.), reducing $10 \%$ by weight, $41 \%$ of volumes and $33 \%$ of air waybills, demonstrating that are significant and positive the results of the actions of Ibama. In addition to benefiting the depositary, freeing usable space in the cargo terminals, the solution of the abandoned loads implies the reduction of damage to the Public Treasury, as well as in reducing potentially harmful accident risks to people and the environment.
\end{abstract}

KEYWORDS: Solid waste. Management. Environmental politics. International trade.

\section{RESUMEN}

La cantidad de residuos sólidos producidos en los aeropuertos es equivalente a los residuos generados en las ciudades pequeñas (5-30 mil residentes), por lo que la gestión de residuos uno de los problemas ambientales más importantes en las instalaciones del aeropuerto. Además de los residuos generados habitual dentro de los aeropuertos, una importante (pero poco conocida) fuente de desechos está asociada con operaciones de comercio exterior: cargas abandonadas o sujetos a penalidad de pérdida (en proceso de confiscación). El objetivo de este trabajo es dar a conocer una iniciativa, en implementación por el Ibama en el Aeropuerto Internacional de Viracopos (Campinas/SP/Brasil), que consiste en la aplicación de la Política Nacional de Residuos Sólidos (Ley brasileña $n^{\circ} 12.305 / 2010$ ) para la reducción y eliminación adecuada de residuos sólidos relacionados a cargas abandonadas en los aeropuertos. De las aproximadamente 1.000 toneladas de cargas abandonadas en Viracopos, fue promovido, en 2014 y 2015, la solución a 35.000 cargas (o 103 ton.), la reducción de $10 \%$ en peso, el 41\% del volumen y el $33 \%$ de las guías aéreas, lo que demuestra que son resultados significativos y positivos de las acciones de Ibama. Además de beneficiar al depositario, liberando espacio valioso en los terminales de carga, la solución de las cargas abandonadas implica la reducción de daños a la Hacienda, así como en la reducción de los riesgos de accidentes potencialmente dañinos para las personas y el medio ambiente.

PALABRAS CLAVE: Residuos sólidos. Gestión. Política ambiental. Comercio exterior. 


\section{INTRODUÇÃO}

\subsection{Aspectos ambientais em aeroportos}

Os aeroportos são geralmente percebidos pelos efeitos ambientais negativos: poluição do ar, poluição sonora, geração de resíduos sólidos e impactos hídricos (consumo de água e geração de esgoto). Geralmente, pouco destaque é dado à gestão de resíduos, em virtude de maior preocupação com o ruído e as emissões das aeronaves. Mas, considerar os resíduos é de suma importância, já que o impacto ambiental é significativo - caso não seja mitigado - e sua destinação implica em elevado custo aos aeroportos (PITT; BROWN; SMITH, 2002).

Em termos de consumo de energia e materiais, bem como geração de resíduos e emissões, um aeroporto pode ser visto como uma cidade pequena ou média. Logo, este tipo de empreendimento causa impactos significativos sobre o volume de resíduos produzidos na região em que está situado. Pitt, Brown e Smith (2002) relatam o caso do Aeroporto Internacional de Dallas/Fort Worth (EUA), um dos maiores do mundo: em 1993, ao transportar cerca de 50 milhões de passageiros, o aeroporto produziu uma quantidade de resíduos que equivale a de uma cidade com 250.000 habitantes. Dados mais atualizados de Carvalho et al. (2013), conforme ilustrado na Figura 1, indicam uma proporção não tão exagerada quanto o estudo anterior: aeroportos com tráfego de 50 milhões de passageiros correspondem a cidades com uma população equivalente de 20 mil pessoas, em termos de uso de água.

Figura 1: Relação entre o tráfego de passageiros nos aeroportos mais importantes do mundo e o equivalente de população, em termos de consumo de água, relativo ao ano de 2010. Cada ponto representa um aeroporto.

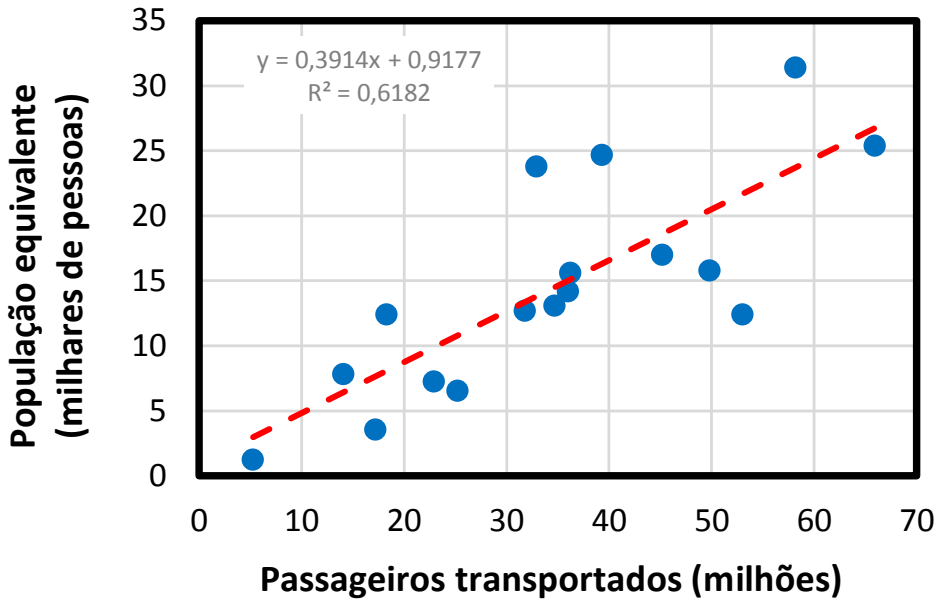

Fonte: elaborado com base em Carvalho et al. (2013).

Além dos resíduos gerados diretamente por passageiros e demais usuários, há considerável parte de resíduos gerados em atividades operacionais, como as atividades de importação de cargas. Entre as mercadorias submetidas a operações de comércio exterior, figura um volume de cargas que são abandonadas pelos interessados nos recintos alfandegados do sítio aeroportuário, intensificando o risco ambiental nestes locais. 


\subsection{Aeroporto Internacional de Viracopos}

O Aeroporto Internacional de Viracopos localiza-se ao sul do município de Campinas/SP, distante $14 \mathrm{~km}$ de seu centro e cerca de $93 \mathrm{~km}$ a noroeste do centro do município de São Paulo. Em atividade desde a década de 30, Viracopos é considerado um dos aeroportos mais importantes do Brasil, seja devido a sua localização privilegiada, em uma região metropolitana de grande concentração industrial no Estado, seja devido à proximidade com as principais rodovias estaduais (Santos Dumont, Bandeirantes, Anhanguera) que favorecem o fácil acesso dos passageiros bem como o rápido fluxo das cargas (WALM, 2008).

Em 2015, Viracopos movimentou 52 mil ton. de cargas na exportação (ABV, 2016a) e 155 mil ton. de cargas na importação (ABV, 2016b) - representando cerca de 33\% de toda carga aérea importada no País; em termos de fluxo de passageiros, em 2015, mais de 10 milhões de pessoas $^{1}$ (ABV, 2016c) passaram pelo Aeroporto. Atualmente em funcionamento, o novo Terminal de Passageiros possui a capacidade de atender 25 milhões de passageiros por ano (ABV, 2016d). Segundo o Plano Diretor do Aeroporto (NACO, 2013), prevê-se, na fase final da ampliação (ano 2042), a capacidade de transportar 75 milhões de passageiros ${ }^{2}$ por ano e 1,4 milhões de toneladas anuais de cargas.

Em relação ao Aeroporto de Viracopos, é possível também obter uma estimativa da população equivalente, seja em termos de consumo de água (Tabela 1), consumo de energia elétrica (Tabela 2) e geração de resíduos sólidos (Tabela 3). Dos dados apresentados nas Tabelas, considera-se tanto a situação atual quanto o cenário de ampliação prevista para o ano de 2042. Constata-se que, na média das estimativas de população equivalente, Viracopos equivale hoje a uma cidade de 15.000 habitantes, podendo equivaler a uma cidade de 105 mil habitantes em 2042. Essa estimativa futura pode não necessariamente se concretizar, visto que a realidade dos aeroportos que hoje movimentam em torno de 70 milhões de passageiros/ano equivalem a cidades com 25 a 30 mil habitantes (conforme mostra a Figura 1).

Tabela 1: Estimativa de população equivalente do Aeroporto de Viracopos, com base no consumo de água

\begin{tabular}{|c|c|c|c|}
\hline \multirow{2}{*}{ Ano } & Aeroporto & Referência & Pop. Equiv. \\
\hline & $\left(\mathrm{m}^{3} / \mathrm{dia}\right)$ & (L/hab/dia) & (hab) \\
\hline 2012 & $1.217^{(\mathrm{a})}$ & \multirow{2}{*}{$162,0^{(b)}$} & 7.512 \\
\hline 2042 & $17.065^{\text {(a) }}$ & & 105.339 \\
\hline
\end{tabular}

Fonte: elaborado com base nos dados de: ${ }^{(a)}$ NACO (2013); e ${ }^{(b)}$ Brasil (2016).

\footnotetext{
${ }^{1}$ Esse valor representa, na média, 27 mil passageiros por dia atualmente. Além dos passageiros, o Aeroporto possui hoje cerca de 9 mil funcionários (NACO, 2013). Ou seja, no total, são 36 mil pessoas circulando por dia.

2 Esse valor representará, no ano de 2042, a média de 200 mil passageiros por dia. Além dos passageiros, no cenário futuro (daqui a 26 anos), o Plano Diretor (NACO, 2013) prevê que Viracopos possuirá cerca de 75 mil funcionários, totalizando 275 mil pessoas circulando por dia no recinto aeroportuário.
} 
Tabela 2: Estimativa de população equivalente do Aeroporto de Viracopos, com base no consumo de energia

\begin{tabular}{cccc}
\hline \multirow{2}{*}{ Ano } & Aeroporto & Referência & Pop. Equiv. \\
\cline { 2 - 4 } & $(\mathrm{MWh})$ & $(\mathrm{kWh} /$ mês/residência) & (hab) \\
\hline 2012 & $30^{\text {(a) }}$ & $167,0^{\text {(b) }}$ & 17.725 \\
\hline 2042 & $195^{\text {(a) }}$ & & 115.658 \\
\hline
\end{tabular}

Fonte: elaborado com base nos dados de: ${ }^{(a)}$ NACO (2013); e ${ }^{(b)}$ Brasil (2015). Obs: considera-se que 1 residência possua 3,3 habitantes.

Tabela 3: Estimativa de população equivalente do Aeroporto de Viracopos, base na produção de resíduos sólidos

\begin{tabular}{ccccc}
\hline \multirow{2}{*}{ Ano } & Aeroporto & Referência & Pop. Equiv. \\
\cline { 2 - 2 } & $(\mathrm{kg} / \mathrm{dia})$ & & $(\mathrm{kg} /$ hab/ano $)$ & (hab) \\
\hline 2012 & $20.178^{\text {(a) }}$ & \multirow{2}{*}{$387,3^{\text {(b) }}$} & 19.001 \\
\hline 2042 & $103.740^{\text {(a) }}$ & & 97.691 \\
\hline
\end{tabular}

Fonte: elaborado com base nos dados de: ${ }^{(a)}$ NACO (2013); ${ }^{\text {(b) }}$ ABRELPE (2014).

\subsection{Resíduos sólidos em Aeroportos}

De acordo com Pitt, Brown e Smith (2002), a gestão de resíduos é uma das questões ambientais mais importantes para os aeroportos. Também denominado de resíduo de serviços de transportes ${ }^{3}$, resíduo aeroportuário é todo aquele sólido ou semissólido que resulta de atividades específicas de origens diversas desenvolvidas dentro das fronteiras dos aeroportos ou a bordo de aeronaves que a eles se destinam (CARRA; CONCEIÇÃO; TEIXEIRA, 2013).

No Brasil, as principais normas legais que tratam sobre o assunto, são: a Resolução CONAMA no 05/1993; a Resolução RDC ANVISA no 56/2008 e a NBR 8.843, que fornecem diretrizes de boas práticas e ações mínimas para cada etapa do gerenciamento de resíduos sólidos em aeroportos, incluindo as obrigações e os responsáveis pelo cumprimento das disposições. Cada normativa estabelece algumas classificações ${ }^{4}$ dos resíduos sólidos. Baseando-se na Resolução RDC ANVISA no 56/2008, Carra, Conceição e Teixeira (2013) apresentam a classificação:

- Grupo A: apresentam risco biológico (gerado a bordo de aeronaves, ambulatórios e terminais de carga);

- Grupo B: apresentam risco químico (gerado em áreas industriais e de manobras, como óleos, lâmpadas de mercúrio e baterias);

- Grupo C: materiais radioativos ou contaminados com radioisótopos;

- Grupo D: resíduo comum;

- Grupo E: perfuro-cortante (lâminas, agulhas, ampolas de vidro e escalpes).

\footnotetext{
${ }^{3}$ Conforme classificado no art. 13, inciso I, alínea "j”, da Lei no 12.305/2010 - Política Nacional de Resíduos Sólidos).

${ }^{4}$ Carra (2011) aborda em detalhes as diferenças entre as classificações.
} 
Segundo Carra, Conceição e Teixeira (2013), além dos resíduos comuns (papel, plástico, resíduos de alimentos, guardanapos etc.) existem resíduos perigosos gerados no aeroporto de grande volume: infectantes (forração de baia do terminal de cargas vivas e resíduos de bordo de aeronaves, ambulatório médico do terminal de passageiros); resíduos com risco químico (lâmpadas fluorescentes, baterias utilizadas em empilhadeiras, óleos, tintas, lubrificantes, pneus inservíveis); resíduos de poda e capina (áreas verdes) e construção civil (entulho).

No Aeroporto Internacional de Viracopos, em 2010 foram geradas aproximadamente 100 toneladas por mês, das quais aproximadamente $97 \%$ correspondem aos resíduos dos Grupos A e $D$, gerados principalmente nos terminais de passageiros e cargas e pelas companhias aéreas a bordo das aeronaves (CARRA; CONCEIÇÃO; TEIXEIRA, 2013). Segundo WALM (2008), geravase em Viracopos aproximadamente 60 toneladas de resíduos/mês em 2008, provenientes das áreas administrativas e operacionais. Assim, na média das fontes supracitadas, pode-se assumir como 80 ton. a quantidade de resíduos sólidos produzidos por mês em Viracopos.

Além dos resíduos inerentes à operação do aeroporto, cabe levantar aqui a geração de um tipo de resíduo pouco conhecido, porém muito relevante, que está associado às operações de comércio exterior (importação e exportação de cargas). Na literatura, pouco é citado ${ }^{5}$ sobre a geração de resíduos que estão relacionados a cargas que são abandonadas nos terminais de cargas. Basicamente, cargas abandonadas são aquelas que chegam ao País por meio da importação e que ficam paradas nos terminais por um período muito prolongado de tempo.

Dentro de um aeroporto, a rotatividade e o fluxo de cargas é processo relativamente rápido, sendo todo o trâmite de importação de cargas encerrado normalmente em questão de dias ou algumas semanas. Mas existem cargas que ficam muitas semanas aguardando a importação, processo que nunca é realizado. Nos casos em que as cargas ficam armazenadas por mais de 3 meses, a legislação aduaneira classifica essas cargas como abandonadas.

Para contextualizar a importância do tema e dar uma noção da dimensão do problema das cargas abandonadas, basta comparar a quantidade de resíduos sólidos comumente gerados nas atividades habituais do Aeroporto de Viracopos com a quantidade de cargas abandonadas que atualmente estão armazenadas neste local: considerando o valor médio de 80 ton./mês de resíduos sólidos produzidos em Viracopos, obtém-se a produção anual de 960 toneladas. Esse valor corresponde quase à quantidade de cargas que acumuladamente foram abandonadas nos últimos 12 anos e que estão armazenadas no antigo Terminal de Cargas do Aeroporto. Em outras palavras, a quantidade de cargas abandonadas hoje equivale à quantidade de resíduos gerados em um ano de funcionamento do Aeroporto de Viracopos, ou, ainda, equivale à produção de resíduos de uma cidade com 15.000 habitantes.

\subsection{Cargas abandonadas ou sujeitas à pena de perdimento}

\footnotetext{
${ }^{5}$ Sobre cargas abandonadas, identificou-se somente o trabalho de Schneider (2004), que apenas cita a questão, porém não fez um levantamento quantitativo da situação.
} 
No Brasil, um dos grandes problemas enfrentados por operadores logísticos de portos e aeroportos consistem nas cargas que são abandonadas nestes recintos. Por configurarem danos ao Erário, aplica-se a pena de perdimento para cargas abandonadas, que são aquelas mercadorias que permanecem em recinto alfandegado sem que o seu despacho de importação seja iniciado no decurso dos seguintes prazos: 90 dias da sua descarga ou 60 dias da data da interrupção do despacho por ação ou omissão do importador ou seu representante.

A partir do abandono (art. 642 do Regulamento Aduaneiro - RA, Decreto no 6.759/2009) a carga fica então sujeita à pena de perdimento (art. 689 do RA), situação que é determinada pela Receita Federal do Brasil (RFB) por meio da lavratura de auto de infração (art. 774 do RA). Feita a triagem e lavrado o auto, a RFB determina a destinação (art. 803 do RA), podendo a carga ser alienada (doada/leiloada), incorporada, inutilizada ou destruída. A Figura 2 apresenta um esquema simplificado das etapas do fluxo de cargas abandonadas segundo o RA.

Figura 2: Fluxograma simplificado do procedimento referente a cargas abandonadas, segundo o Reg. Aduaneiro.

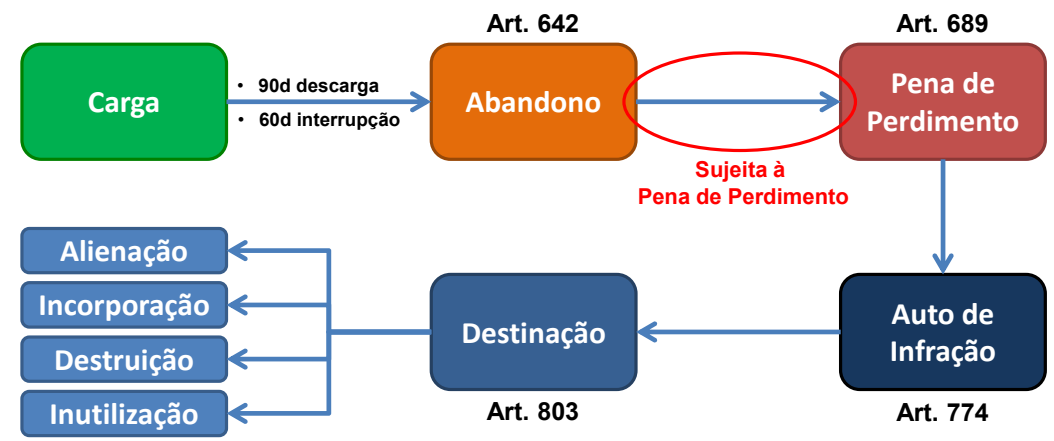

Fonte: elaboração própria.

Com o barateamento do frete aéreo nos últimos anos, naturalmente houve um incremento do volume de cargas importadas e, proporcionalmente, também de cargas abandonadas. Desconhecimento do recebimento pelo importador, envio não solicitado de amostras/brindes, falhas de comunicação, falta no controle dos pedidos (rastreio/recebimento de cargas), inação dos importadores, mesmo quando cientes do desembarque de cargas de sua responsabilidade, são possíveis causas de cargas diariamente abandonadas nos recintos alfandegados do Brasil.

O abandono de mercadorias tem sido apontado como a principal causa de prejuízos significativos no uso do valioso espaço nos Terminais de Carga, devido ao grande volume ocupado em detrimento das cargas que trazem receita aos operadores. Ademais, as cargas abandonadas representam o potencial de tornarem-se um passivo ambiental (resíduo sólido), constituindo fontes potenciais de risco ao meio ambiente e à saúde humana. Além dos riscos ambientais, um eventual vazamento do conteúdo dessas cargas (em especial os produtos perigosos), pode ocasionar grandes acidentes. Nesse cenário extremo, são significativos os impactos econômicos relativos ao funcionamento do aeroporto e, principalmente, impactos ocupacionais e sociais - no caso de Viracopos pode atingir cerca de 30 mil pessoas, incluindo funcionários e passageiros, que frequentam diariamente este Aeroporto, sem contar as milhares de pessoas que moram no entorno do sítio aeroportuário. 


\section{OBJETIVOS}

Em conjunto com o Grupo de Mercadorias Abandonadas da Receita Federal do Brasil (GMAB/RFB) e a concessionária Aeroportos Brasil Viracopos (ABV), a Unidade Avançada do Ibama no Aeroporto de Viracopos (Ibama UA-VCP) ${ }^{6}$ deu início à implementação de ações e procedimentos inéditos com o intuito de promover a solução de cargas abandonadas no Aeroporto de Viracopos. O objetivo principal deste trabalho é relatar a iniciativa que consiste em aplicar a legislação ambiental - com base em princípios ${ }^{7}$ da Política Nacional de Resíduos Sólidos (PNRS), Lei no 12.305/2010 - para a redução e correta destinação de resíduos sólidos relacionados a cargas abandonadas em aeroportos.

\section{METODOLOGIA}

\subsection{Diagnóstico da situação e quantificação do problema}

Estima-se $^{8}$ que somente pequena parte $(0,05 \%)$ das cargas importadas é abandonada. Entretanto, considerando cerca de 1,2 milhão de toneladas que foram importadas entre 2004 e 2012 em Viracopos, quase 1.000 toneladas de cargas abandonadas foram acumuladas no antigo Terminal

de Cargas

\footnotetext{
${ }^{6}$ Neste recinto, o Ibama UA-VCP tem fortalecido seus esforços na fiscalização, permanente e in loco, em operações de comércio exterior e controle de ilícitos ambientais transnacionais.

${ }^{7}$ Alguns princípios da PNRS: a proteção da saúde pública e da qualidade ambiental; a prevenção e a precaução de danos ambientais; a redução do volume e da periculosidade dos resíduos perigosos; a articulação entre as diferentes esferas do poder público, e destas com o setor empresarial, com vistas à cooperação técnica e financeira para a gestão integrada de resíduos sólidos; e a responsabilidade compartilhada pelo ciclo de vida dos produtos.

${ }^{8}$ Com base em estatísticas fornecidas pela ABV.
} 
Figura 3), o qual possui uma área de aproximadamente $7.300 \mathrm{~m}^{2}$ (o equivalente a 1 campo oficial de futebol). Tais cargas perfazem cerca de 70.000 volumes, dos quais 3.800 volumes são produtos perigosos, com peso aproximado de 45 toneladas $^{9}$.

\footnotetext{
${ }^{9}$ Em 2012, o montante total de cargas abandonadas e apreendidas, com valor estimado em $\mathrm{R} \$ 360$ milhões, ocupava $70 \%$ da área útil destinada à armazenagem no Terminal de Cargas (TECA) do aeroporto (CORREIO POPULAR, 2012).
} 
Figura 3: Imagem de satélite do Aeroporto Internacional de Viracopos (à esquerda) e detalhe da área de armazenagem de cargas abandonadas (à direita).

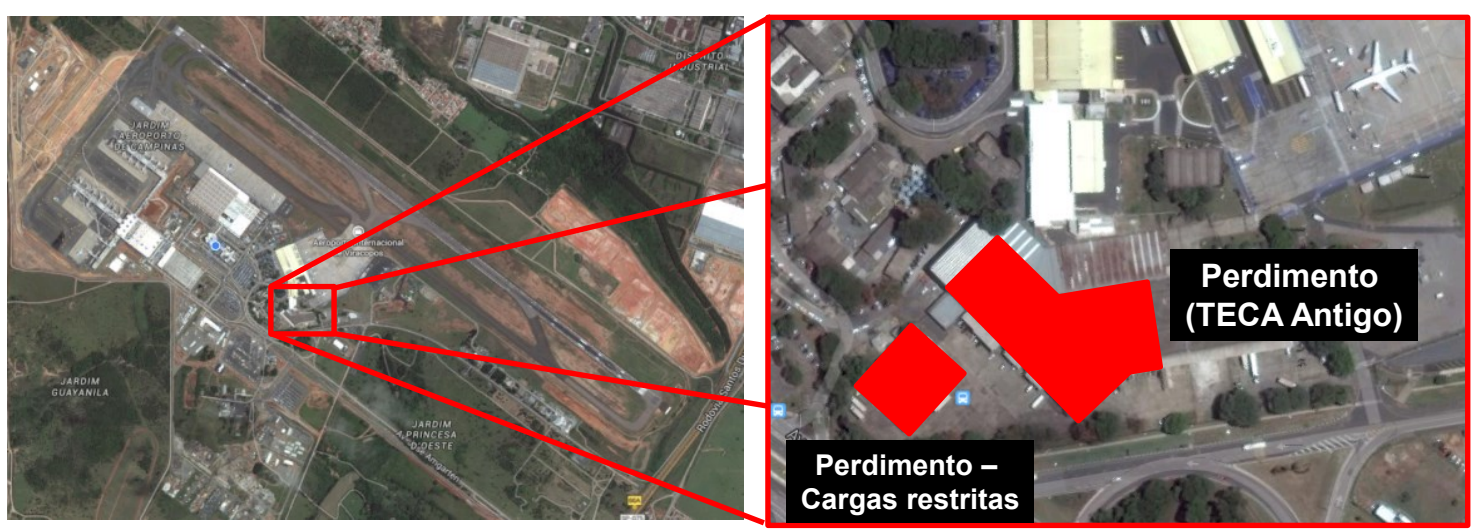

Fonte: elaboração própria, com uso de imagens do Google Maps.

\subsection{Respaldo legal e proposição de solução do problema}

Fundamentado no art. 20 da Resolução CONAMA no 5/1993 e na Resolução CONAMA no 2/1991, o Ibama tem a competência de adotar as medidas necessárias para facilitar a solução final de problemas afetos às cargas abandonadas. A Política Nacional de Resíduos Sólidos (Lei no 12.305/2010) corrobora com as Resoluções do CONAMA, imputando ao responsável pela carga (importador, transportador aéreo ou depositário) as ações de prevenção, controle, tratamento e disposição final dos resíduos gerados pelas cargas abandonadas.

Após análise da situação apresentada e da legislação ambiental, elaborou-se uma proposta piloto de fiscalização. Assim, em trabalho pioneiro, o Ibama UA-VCP definiu e implantou procedimentos para a redução dos impactos causados pelo abandono de cargas em Viracopos: resumidamente, o processo inicia-se com a inspeção da carga abandonada, identificação do conteúdo e do responsáve $\mathrm{l}^{10}$, o qual, então, posteriormente é notificado para apresentar uma solução para tal carga. $O$ não atendimento à notificação é passível de aplicação de multa diária, conforme o art. 64, §1으, do Decreto no 6.514/2008. A infração administrativa com enquadramento no referido artigo possui correspondência na Lei de Crimes Ambientais (Lei no 9.605/1998), cabendo a comunicação ${ }^{11}$ de crime ao Ministério Público Federal (MPF).

Após notificado ${ }^{12}$, o responsável pela carga deve optar por uma das soluções da carga

\footnotetext{
${ }^{10}$ A iniciativa tem como público-alvo direto os responsáveis pelas cargas abandonadas. Em primeiro nível de responsabilidade, entende-se que os importadores são os principais motivadores da chegada de cargas ao país, uma vez que, salvo exceções (amostras/brindes), as cargas são despachadas na origem mediante alguma compra ou pedido que foi realizado no Brasil. Os transportadores aéreos e depositários também são considerados como corresponsáveis pelas cargas.

${ }^{11}$ A comunicação de crime é acompanhada de toda documentação pertinente, bem como histórico de infrações do autuado. Paralelamente à apuração da ocorrência da infração administrativa ambiental, pode ocorrer a persecução penal pelo MPF sobre o cometimento de crime ambiental pelo infrator.

${ }^{12}$ As empresas importadoras, antes de serem notificadas ou autuadas pelo lbama, também podem agir preventivamente para a redução da quantidade de cargas que são abandonadas, bastando orientar os
} 
abandonada: 1. Retomada do despacho aduaneiro; 2. Devolução; ou 3. Destinação final ambientalmente adequada (destruição, incineração, trituração etc.). As cargas abandonadas selecionadas para aplicação e implementação de rotina e fluxograma de ações seguem as etapas descritas no fluxograma da Figura 4.

Figura 4: Fluxograma simplificado das etapas/procedimentos para solução de cargas abandonadas

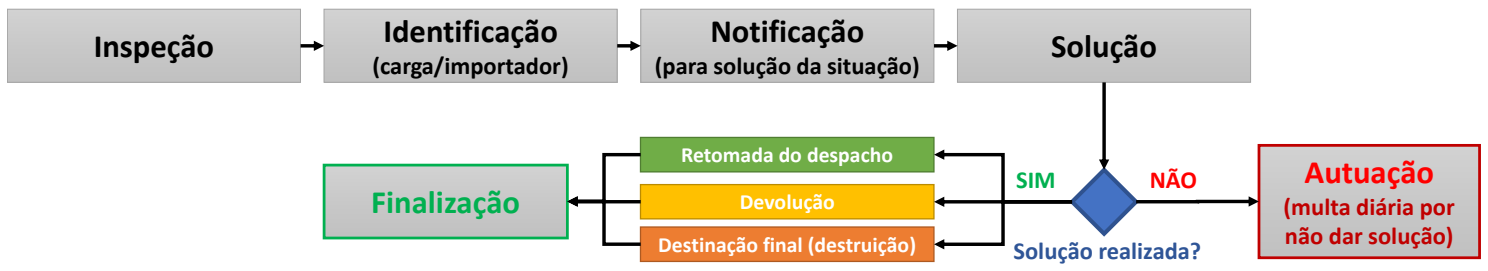

Fonte: elaboração própria.

Qualquer carga abandonada, com o potencial de se tornar um resíduo, pode ser alvo de fiscalização, sendo prioritárias as cargas com substâncias químicas e perigosas de maior volume e/ou peso, pois oferecem potencialmente maiores riscos ambiental e ocupacional.

\section{RESULTADOS}

Centenas de cargas inspecionadas em força-tarefa da RFB, ABV e órgãos anuentes (Anvisa, Ibama e MAPA) foram encaminhadas para destruição em 2014 e 2015, dando solução para aproximadamente 103 toneladas de cargas abandonadas. Desse total, cerca de 15,4 toneladas de cargas $^{13}$ tiveram a solução executada por empresas importadoras que foram notificadas pelo Ibama. Do total das cargas cujos importadores foram notificados, as soluções dadas foram: $5 \%$ de devolução, $10 \%$ de autuação ${ }^{14}, 35 \%$ de destinação final ambientalmente adequada (destruição) e $50 \%$ de retomada do despacho

\footnotetext{
despachantes aduaneiros e os agentes de carga a aumentar o controle dos pedidos e do rastreio de cargas importadas e alertar os fornecedores sobre o não envio de cargas e amostras sem aviso prévio.

${ }^{13}$ Esse quantitativo representa resultados diretos, ou seja, o peso das cargas registradas em termos de inspeção do Ibama. Entretanto, destaca-se que a aplicação da legislação ambiental tem impacto muito maior no total de cargas destinadas, tendo como principal resultado a mudança de paradigma na comunidade aeroportuária. Nota-se um novo comportamento dos responsáveis por cargas abandonadas, os quais têm adotado postura proativa na retirada de suas cargas ou, até mesmo, preventiva, evitando o abandono.

${ }^{14}$ Das cargas que tiveram autuações, cabe destacar que as multas lavradas forçaram diversas empresas a tomar providências e buscar alguma das soluções. Nos casos não resolvidos mesmo após aplicação de multa, o Ministério Público Federal tem instaurado ações civis públicas que estão motivando inquéritos criminais da Polícia Federal.
} 
Figura 5). 
Figura 5: Soluções de cargas abandonadas cujos importadores foram notificados pelo Ibama UA-VCP.

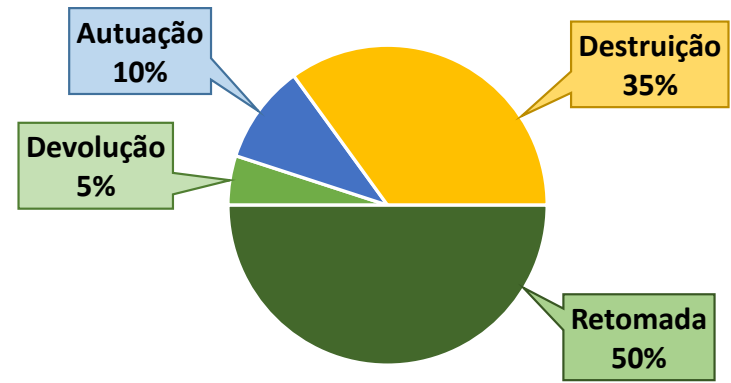

Fonte: elaboração própria, com dados do Ibama UA-VCP.

Em 2014 e 2015, 35.000 cargas (ou 103 ton.) foram resolvidas (Tabela 4), reduzindo 10\% do peso, $41 \%$ dos volumes e $33 \%$ dos conhecimentos aéreos (Air Waybill - AWB) (Figura 6) do passivo total. Para as cargas restritas (produtos perigosos), a redução foi de $38 \%$ do peso, $42 \%$ dos volumes

e

$57 \%$

dos

AWB 
Figura 7).

Tabela 4: Cargas solucionadas

\begin{tabular}{|c|c|c|c|c|c|c|}
\hline \multirow{2}{*}{ Ano } & \multicolumn{2}{|c|}{ Peso (toneladas) } & \multicolumn{2}{|c|}{ Volumes (quantidade) } & \multicolumn{2}{|c|}{ AWB (quantidade) } \\
\hline & VCP & Ibama & VCP & Ibama & VCP & Ibama \\
\hline 2014 & 30 & 1,5 & 5.116 & 111 & $\approx 16.000$ & 37 \\
\hline 2015 & 73 & 13,9 & 12.691 & 214 & $\approx 19.000$ & 202 \\
\hline 2016 & \multicolumn{2}{|c|}{$29^{(\mathrm{a})}$} & - & - & - & - \\
\hline
\end{tabular}

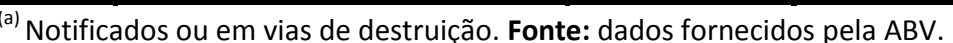

Figura 6: Redução de pesos, volumes e AWB dos totais de cargas abandonadas em Viracopos.

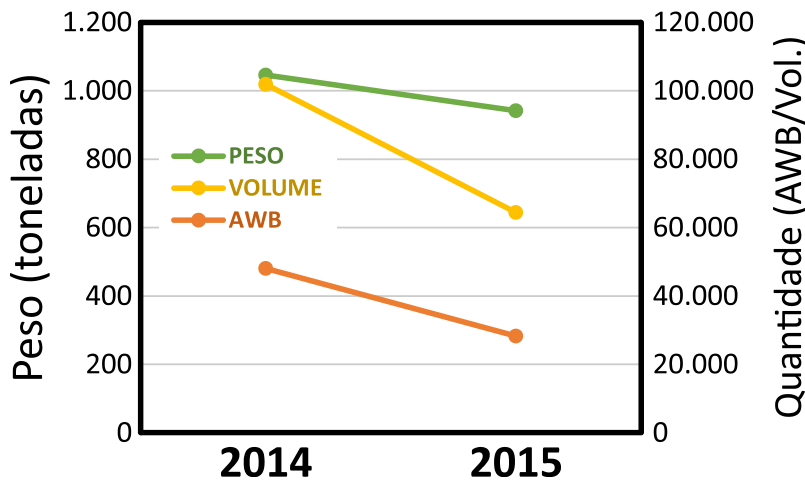

\begin{tabular}{|c|c|}
\hline \multicolumn{2}{|c|}{ Cargas (total) } \\
\hline & Redução \\
\hline Peso & $10 \%$ \\
\hline Volumes & $41 \%$ \\
\hline AWBs & $33 \%$ \\
\hline
\end{tabular}

Fonte: elaboração própria, com dados fornecidos pela ABV. 
Figura 7: Redução de pesos, volumes e AWB das cargas restritas abandonadas em Viracopos.
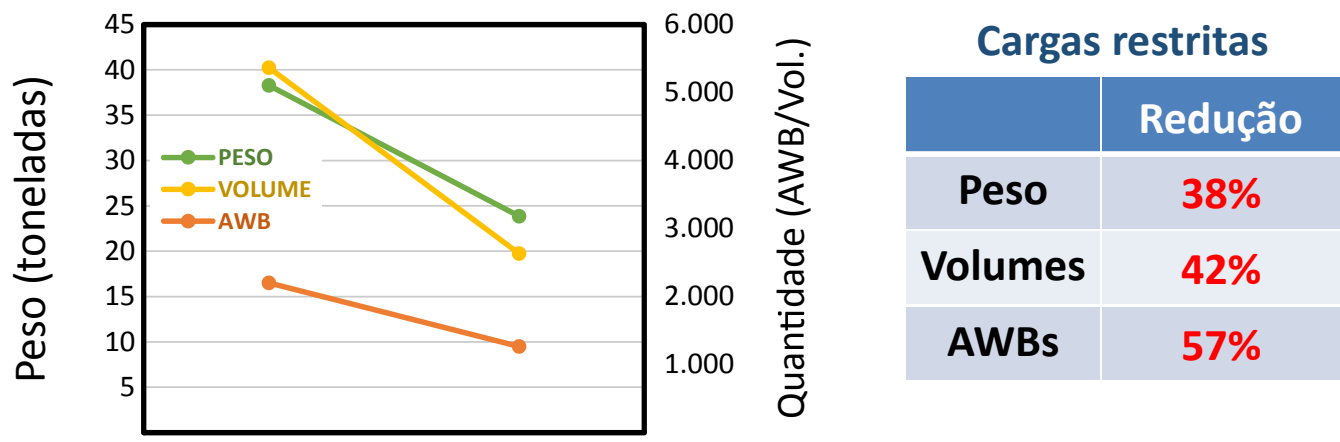

Fonte: elaboração própria, com dados fornecidos pela ABV.

Pelos dados apresentados, nota-se que são expressivos e positivos os resultados das ações do Ibama no casos de cargas abandonadas em Viracopos, ressaltando que esta importante iniciativa já está sendo considerada como referência para replicação em outros recintos alfandegados (aeroportos, portos e portos secos).

Usando estatísticas de Viracopos (0,05\% das cargas importadas são abandonadas), pode-se obter uma estimativa expedita da quantidade de cargas abandonadas no Brasil: nos aeroportos ( 2.725 ton.) e portos (2,7 milhões de ton.), conforme apresenta a Tabela 5.

Tabela 5: Situação estimada ${ }^{(1)}$ de cargas abandonadas no Brasil

\begin{tabular}{|c|c|c|}
\hline $\begin{array}{l}\text { Recinto } \\
\text { alfandegado }\end{array}$ & $\begin{array}{l}\text { Volume de Importação de cargas } \\
\text { (ton) }\end{array}$ & $\begin{array}{c}\text { Cargas abandonadas (ton) } \\
(0,05 \% \text { do total })^{(1)}\end{array}$ \\
\hline Portos $^{(2)}$ & $\begin{array}{c}3.537 .066 .765 \\
\text { (3,5 bilhões) }\end{array}$ & $\begin{array}{c}2.720 .821 \\
(2,7 \text { milhões })\end{array}$ \\
\hline Aeroportos $^{(3)}$ & $\begin{array}{c}3.568 .673 \\
\text { (3,5 milhões) }\end{array}$ & 2.745 \\
\hline Aeroporto de Viracopos ${ }^{(3)}$ & $\begin{array}{c}1.180 .116 \\
\text { (1,2 milhões) }\end{array}$ & 908 \\
\hline
\end{tabular}

Com o intuito de replicação da iniciativa em outros recintos, tem sido discutidas e definidas padronizações de procedimentos para as unidades integrantes do Grupo de Trabalho do Comércio Exterior no Ibama de SP (GT-Comex Ibama/SP). Além disso, membros do GT-Comex Ibama/SP têm participado de reuniões da Comissão Nacional de Autoridades Aeroportuárias (CONAERO), em especial no "GT Cargas", onde essa iniciativa do Ibama vem sendo apresentada e positivamente recebida.

De um modo geral, a solução das cargas abandonadas beneficia o depositário, liberando espaço útil nos terminais de cargas e também reduz os recursos (humanos e financeiros) que a autoridade aduaneira (RFB) dedicaria à apuração de infrações e destinação das cargas. Por fim, são reduzidos os riscos de dano ao meio ambiente, às pessoas (trabalhadores, passageiros e 
moradores no entorno) e ao Erário.

A situação da quantidade e tipos de cargas abandonadas no Aeroporto Internacional de Viracopos é ilustrada por meio da Figura 8, onde são apresentadas fotos de cargas no Terminal de Perdimento e fotos de procedimento de destruição (incineração) de cargas.

Figura 8: (A e B) - Cargas abandonadas no Terminal de Perdimento (antigo Terminal de Cargas) do Aeroporto Internacional de Viracopos; (C) - Amostra da diversidade de "cargas restritas", ou seja, contendo produtos químicos perigosos diversos; (D) - Processo de destinação final ambientalmente adequada (destruição: incineração) de cargas abandonadas consideradas como resíduos sólidos.
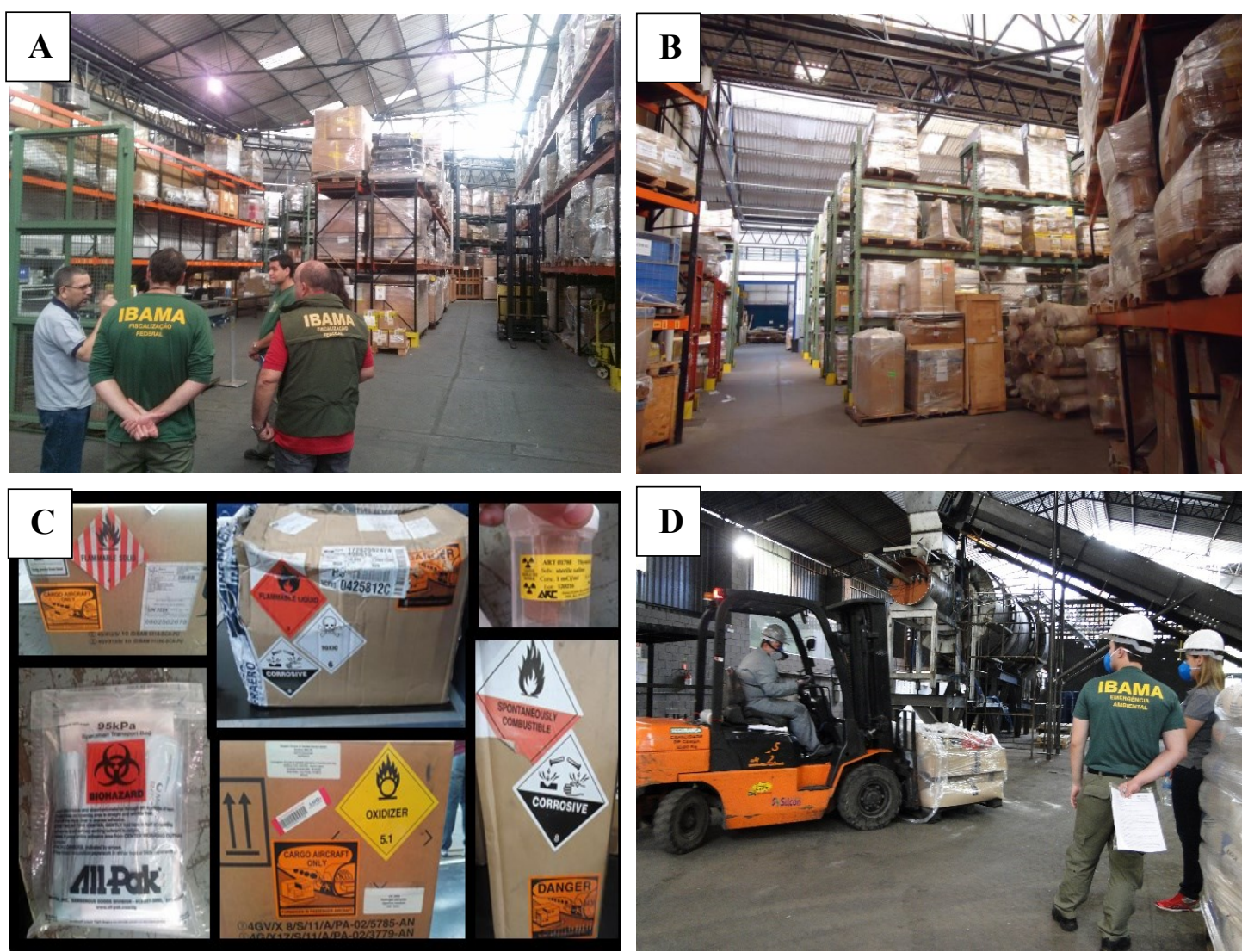

Fonte: fotos do Ibama UA-VCP.

\section{CONCLUSÃO}

A iniciativa tem se demonstrado como um eficiente conjunto de procedimentos técnicooperacionais e administrativos que realmente está resolvendo um enorme passivo e reduzindo a quantidade de novas cargas abandonadas no Aeroporto Internacional de Viracopos. Ainda, se tomada como piloto, a iniciativa pode ser replicada e contribuir para a resolução de um antigo problema (cargas abandonadas) existente em praticamente todos os recintos alfandegados (aeroportos, portos e portos secos) do Brasil. Em termos ambientais, a iniciativa apresenta uma solução eficaz das cargas que recentemente estão sendo abandonadas e também do enorme passivo de cargas que foram sendo abandonadas e acumuladas ao longo 
de anos no sítio aeroportuário.

Em termos econômicos, as demonstrações financeiras (ABV, 2015) mostram que $61 \%$ da receita de Viracopos é proveniente da operação de cargas. Ou seja, em 2014, dos cerca de R\$ 460 milhões da receita operacional, as operações do terminal de cargas representaram $R \$ 281$ milhões da fonte de arrecadação. As operações aeronáuticas (transporte de passageiros) representaram $22 \%$ da receita ( $\mathrm{R} \$ 101$ milhões) e as receitas comerciais representaram $17 \%$ (R\$ 78 milhões).

Uma vez que o transporte de mercadorias constitui quase $2 / 3$ (dois terços) da receita obtida pelo aeroporto, fica comprovada que as medidas para a desobstrução do terminal de cargas melhoram a eficiência do setor em Viracopos. A retirada de cargas abandonadas representou, em 2015, uma arrecadação adicional na ordem de milhões de reais, oriunda de tarifas de armazenagem e de destruição pagas às expensas dos importadores notificados pelo Ibama. Além disso, a completa liberação da atual área de armazenamento das cargas abandonadas $\left(7.300 \mathrm{~m}^{2}\right)$ eliminará uma considerável fonte de riscos ambientais e ocupacionais, bem como permitirá a utilização futura do espaço para aumento das receitas comerciais.

Por fim, a iniciativa representa uma importante quebra de paradigma no comércio exterior. Por parte dos importadores, despachantes e de outros profissionais intervenientes na cadeia logística de cargas submetidas a operações de comércio exterior existe, infelizmente, uma cultura de "deixar cair no perdimento que a RFB resolve depois". Assim, o Ibama apresenta esse importante mecanismo de responsabilização, que exige a tomada de atitude para a destinação adequada da carga. A permanência na situação de abandono, decorrente da omissão ou inação do responsável, caracteriza infração passível de sanção administrativa, seguida de comunicação para apuração de crime ambiental. A aplicação da legislação ambiental tem também gerado ação dissuasória, reduzindo o quantitativo de novas cargas abandonadas.

\section{AGRADECIMENTOS}

Os autores agradecem as pessoas sem as quais toda a iniciativa não teria sido possível: servidores da Unidade Avançada do Ibama no Aeroporto Internacional de Viracopos - UA-VCP, que são igualmente autores deste artigo, mas que não puderam assim constar devido a restrições do formato (Alexandre Duarte, Cláudio Cazal, Diego Zanini, Edson Tsuhako, Erli de Abreu, Fernanda Mecabô, Fernanda Wick, Helder de Santanna, José Reato, Karina Piria, Rosilaine Gonçalves), servidores do Grupo de Mercadorias Abandonadas da Receita Federal do Brasil - GMAB/RFB de Viracopos (Adriana Sampaio, Maria Fátima, Tereza Rolim, Terezinha de Sousa); equipe da concessionária Aeroportos Brasil Viracopos - ABV (Bruna Pires, Flávio Bento, Jorge Lobarinhas, Marina Giffu, Ricardo Luize); servidores da Superintendência do Ibama em SP - SUPES-SP [Cinthia Masumoto, Fernando Scavassin, Lucila Claudia, Mariana Nakashima, Marilda Heck, Murilo Reple, Paulo Aredes, Walter de Faria (In memoriam)]; servidores da 
Unidade Avançada do Ibama no Aeroporto Internacional de Guarulhos - UA-GRU (Daniel Visciano, Felipe Seino, Filipe Commetti); servidores da Diretoria de Qualidade Ambiental DIQUA - Ibama Sede (Fernando Marques, Gilberto Werneck); servidores da Diretoria de Proteção Ambiental - DIPRO - Ibama Sede (Luciano Evaristo, Jair Schmitt).

\section{REFERÊNCIAS}

ABRELPE. Panorama dos resíduos sólidos no Brasil - 2014. São Paulo: ABRELPE, 2014. Disponível em: <http://www.abrelpe.org.br/Panorama/panorama2014.pdf>. Acesso em: 12 mai. 2016.

ABV. Aeroporto Brasil Viracopos. Acompanhamento da Carga Exportada - Peso Recebido (Ton) - 2014-2016. $2016 \mathrm{~b}$. Disponível em: <http://www.viracopos.com/cargas/dados-movimentacao/>. Acesso em: 12 mai. 2016.

ABV. Aeroporto Brasil Viracopos. Acompanhamento da Carga Importada - Peso Recebido (Ton) - 2014/2016. 2016a. Disponível em: <http://www.viracopos.com/cargas/dados-movimentacao/>. Acesso em: 12 mai. 2016.

ABV. Aeroporto Brasil Viracopos. Aeroporto de Viracopos transfere voos domésticos para novo terminal de passageiros. 19 abr., 2016d. Disponível em: <http://www.viracopos.com/institucional/imprensa/19-04-2016aeroporto-de-viracopos-transfere-voos-domesticos-para-novo-terminal-de-passageiros.html>. Acesso em: 12 mai. 2016.

ABV. Aeroporto Brasil Viracopos. Demonstrações Financeiras - 2014. 2015. Disponível em: <http://www.viracopos.com/institucional/governanca-corporativa/>. Acesso em: 12 mai. 2016.

ABV. Aeroporto Brasil Viracopos. Resumo de Movimentação Aeroportuaria (RMA) de 2015. Movimento Operacional do Aeroporto de Viracopos Janeiro a Dezembro de 2015. 2016c. Disponível em: <http://www.viracopos.com/institucional/estatisticas/>. Acesso em: 12 mai. 2016.

ANTAQ. Agência Nacional de Transportes Aquaviários. Estatísticas - Anuários. 2015. Disponível em: <http://www.antaq.gov.br/PORTAL/Estatisticas_Anuarios.asp\#>. Acesso em: 12 mai. 2016.

BRASIL. Ministério das Cidades. Secretaria Nacional de Saneamento Ambiental - SNSA. Sistema Nacional de Informações sobre Saneamento: Diagnóstico dos Serviços de Água e Esgotos - 2014. Brasília: SNSA/MCIDADES, 2016. 212 p. Disponível em: <http://www.snis.gov.br/diagnostico-agua-e-esgotos/diagnostico-ae-2014>. Acesso em: 12 mai. 2016.

BRASIL. Ministério de Minas e Energia. Empresa de Pesquisa Energética (EPE). Anuário Estatístico de Energia Elétrica 2015 - ano base 2014. Rio de Janeiro: EPE/MME, 2015. Disponível em: <http://www.epe.gov.br/AnuarioEstatisticodeEnergiaEletrica/Forms/Anurio.aspx>. Acesso em: 12 mai. 2016.

CARRA, Thales Andrés. Metodologia para avaliação de desempenho ambiental em aeroportos e sua aplicação no Aeroporto Internacional de Viracopos, Campinas (SP). Dissertação (mestrado) - Universidade Estadual Paulista, Instituto de Geociências e Ciências Exatas - Rio Claro: 2011.

CARRA, Thales Andrés; CONCEIÇÃO, Fabiano Tomazini da; TEIXEIRA, Bruno Bernardes. Indicadores para a gestão de resíduos sólidos em aeroportos e sua aplicação no Aeroporto Internacional de Viracopos, Campinas, São Paulo. Engenharia Sanitária e Ambiental, v.18, n.2, abr/jun 2013, p. 131-138. [DOI: http://dx.doi.org/10.1590/S141341522013000200005]

CARVALHO, Isabella de Castro; et al. Sustainable airport environments: A review of water conservation practices in airports. Resources, Conservation and Recycling, v. 74, p. 27-36, 2013.2 [DOI: http://dx.doi.org/10.1016/j.resconrec.2013.02.016]

CORREIO POPULAR. Cargas perdidas lotam Viracopos. 08/22/2012. Disponível em: <http://correio.rac.com.br/_conteudo/2012/11/capa/campinas_e_rmc/8984-cargas-perdidas-lotamviracopos.html>. Acesso em: 12 mai. 2016.

INFRAERO. Anuário Estatístico Operacional. 2015.2 Disponível em: <http://www.infraero.gov.br/index.php/br/estatistica-dos-aeroportos.html>. Acesso em: 12 mai. 2016.

NACO. Netherlands Airport Consultants B.V. Plano Diretor Aeroporto Internacional de Viracopos. Sumário 
Executivo. Campinas, 2013.

PITT, Michael; BROWN, Andrew; SMITH, Andrew. Waste management at airports. Facilities, v. 20, n. 5/6, p. 198207, 2002. [DOI: http://dx.doi.org/10.1108/02632770210426684]

SCHNEIDER, Soraia Cristina Ribas Fachini. Gerenciamento de resíduos sólidos em aeroportos estudo de caso Aeroporto Internacional Salgado Filho. Dissertação (Mestrado) - UNIVERSIDADE FEDERAL DE SANTA CATARINA CIÊNCIAS EM ENGENHARIA AMBIENTAL - FLORIANÓPOLIS: 2004.

WALM. Engenharia e Tecnologia Ambiental. EIA - Estudo de Impacto Ambiental: Ampliação do Aeroporto Internacional de Viracopos/Campinas - SP. Volume I - Textos. São Paulo, 2008. 